CrossMark $\quad$ Open Medicine Journal

\title{
LETTER
}

\section{Inhibition of Renal Fibrosis and Glomerular Injury by Sacubitril/Valsartan, a Combination Angiotensin Receptor Blocker and Neprilysin Inhibitor, in a Salt-Sensitive Hypertensive Model Using Angiotensin 1 Receptor Knockout Mice: The Contribution of Non-Angiotensin Blocking Effects to Renal Protection}

Rei Otsu', Yoshiaki Taniyama ${ }^{1,2, *}$, Fumihiro Sanada ${ }^{1}$, Jun Muratsu ${ }^{1,2}$, Kana Shibata ${ }^{1}$, Tatsuya Fujikawa $^{1}$, Kanako May Brule ${ }^{1}$, Hideo Shimizu ${ }^{1}$, Hiromi Rakugi ${ }^{2}$ and Ryuichi Morishita ${ }^{1}$

${ }^{\text {I}}$ Department of Clinical Gene Therapy, Osaka University Graduate School of Medicine, 2-2 Yamadaoka, Suita, Osaka 565-0871, Japan

${ }^{2}$ Department of Geriatric and General Medicine, Osaka University Graduate School of Medicine, Suita, Osaka 565-0871, Japan

Received: October 19, 2018

Revised: November 17, 2018

Accepted: November 20, 2018

Abstract:

\section{Introduction:}

"Aldosterone breakthrough," which is observed in patients receiving long term treatment with angiotensin blockade, is strongly associated with the increased risk of a declining glomerular filtration rate through the profibrotic actions of aldosterone. Sacubitril/valsartan is a newly created combination medicine (the angiotensin receptor blocker valsartan and the neprilysin-inhibitor sacubitril). Therefore, sacubitril/valsartan should have additional organ-protective actions besides the angiotensin blockade.

\section{Methods:}

In this study, we examined the renal protective effect of sacubitril/valsartan in a salt-sensitive hypertension model using angiotensin II type 1a receptor (AT1aR) knockout mice. An oral administration of $1 \% \mathrm{NaCl}$ solution with sacubitril/valsartan $(30$ or 60 $\mathrm{mg} / \mathrm{kg} /$ day) or valsartan ( 15 or $30 \mathrm{mg} / \mathrm{kg} /$ day) alone beginning 7 days before administration of aldosterone was examined in an aldosterone infusion AT1R knockout mouse model as an aldosterone breakthrough model.

\section{Results / Conclusion:}

A significant decrease in Blood Pressure (BP) was observed in the sacubitril/valsartan group compared to the valsartan group under low and high doses. In addition, the pathological analysis of the kidney for glomerular fibrosis by Sirius red staining and for injury by PAS staining demonstrated significant reductions accompanied by a significant reduction in TGF- $\beta$ in the sacubitril/valsartan group compared to the valsartan group. Overall, sacubitril/valsartan, which has the dual actions of the AT1R blockade and neprilysin inhibition, may have additional clinical values for the treatment of hypertensive patients with aldosterone breakthrough.

Keywords: Aldosterone breakthrough, Sacubitril/valsartan, Chronic kidney disease, Renal fibrosis, Glomerular injury, Angiotensin.

\section{INTRODUCTION}

Angiotensin (Ang) II type 1 Receptor (AT1R) Blockers (ARBs) have been widely used for the treatment of

\footnotetext{
* Address correspondence to this author at the Department of Clinical Gene Therapy, Osaka University Graduate School of Medicine, 2-2 Yamadaoka, Suita, Osaka 565-0871, Japan, Tel: +81-6-6210-8351, Fax: +81-6-6210-8359, E-mail taniyama@cgt.med.osaka-u.ac.jp
} 
hypertension and hypertension-related cardiovascular end-organ damage [1]. However, plasma aldosterone levels are known to be elevated in a subset of patients despite Ang II blockade therapy. This phenomenon, which is known as 'aldosterone breakthrough', is strongly associated with an increased risk of a declining glomerular filtration rate through the profibrotic actions of aldosterone [2]. Recently, Sacubitril/Valsartan (Sac/Val), comprising the neprilysin inhibitor (sacubitril) and the ARB valsartan, as a so-called Angiotensin Receptor-Neprilysin Inhibitor (ARNI), has been used clinically [3]. Neprilysin is a ubiquitous enzyme that is responsible for the breakdown of many vasoactive peptides, including the biologically active Natriuretic Peptides (NPs), adrenomedullin, substance P, bradykinin, ET-1, and angiotensin. Sac/Val is a crystalline compound composed of both the ARB valsartan and the neprilysin-inhibitor prodrug sacubitril, which dissociates into its component parts after ingestion. In terms of its impact on global mortality and morbidity in a heart failure (PARADIGM-HF) trial, Sac/Val significantly reduced cardiovascular and all-cause mortality, as well as Heart Failure (HF) hospitalization, in patients with HF with reduced Ejection Fraction (HFrEF) compared with the Angiotensin-Converting Enzyme Inhibitor (ACEI) enalapril [4]. Based on these clinical trials, in patients with HF, Sac/Val seems to be superior for ACEI/ARB. However, it is still unclear how Sac/Val prevented organ injury despite the Ang II blockade. To evaluate molecular mechanisms in how Sac/Val exhibited organ-protective actions, we focused on aldosterone breakthrough with long-term renin-angiotensin inhibition. To examine the effects of $\mathrm{Sac} / \mathrm{Val}$ in the aldosterone breakthrough model, we employed a renal fibrosis model induced by aldosterone and $1 \%$ $\mathrm{NaCl}$ treatment in ATlaR-KO mice [5, 6].

Overall, in this study, we demonstrated the inhibition of renal fibrosis and glomerular injury by Sac/Val, independent from the Ang II blockade.

\section{METHODS}

\subsection{AT1aR Deficient Mouse Model With Salt Sensitive Hypertension}

Mice were divided into 6 groups: (1) nontreatment; (2) aldosterone infusion $(0.15 \mu \mathrm{g} / \mathrm{hour})+1 \% \mathrm{NaCl}$ treatment; (3) valsartan low dose, aldosterone infusion $(0.15 \mu \mathrm{g} /$ hour $)+1 \% \mathrm{NaCl}+$ valsartan $(15 \mathrm{mg} / \mathrm{kg} / \mathrm{day})$ treatment; (4) valsartan high dose, aldosterone infusion $(0.15 \mu \mathrm{g} /$ hour $)+1 \% \mathrm{NaCl}$ and valsartan $(30 \mathrm{mg} / \mathrm{kg} / \mathrm{day})$ treatment; (5) $\mathrm{Sac} / \mathrm{Val}$ low dose, aldosterone infusion $(0.15 \mu \mathrm{g} / \mathrm{hour})+1 \% \mathrm{NaCl}+\mathrm{Sac} / \mathrm{Val}(30 \mathrm{mg} / \mathrm{kg} / \mathrm{day}=$ valsartan $15 \mathrm{mg}$ with NEPi $15 \mathrm{mg}$ ) treatment; and (6) Sac/Val high dose, aldosterone infusion $(0.15 \mu \mathrm{g} / \mathrm{hour})+1 \% \mathrm{NaCl}+\mathrm{Sac} / \mathrm{Val}(60$ $\mathrm{mg} / \mathrm{kg} /$ day = valsartan $30 \mathrm{mg}$ with NEPi $30 \mathrm{mg}$ ) treatment (Supplement 1). Each group contained $10 \mathrm{mice}$.

We started an oral administration of $1 \% \mathrm{NaCl}$ solution and $\mathrm{Sac} / \mathrm{Val}$ or valsartan 7 days before aldosterone (FUJIFILM Wako Pure Chemical Corporation, Osaka, Japan) infusion using an osmotic pump (model 1002; Alzet, DURECT Corporation, CA, USA). Aldosterone infusion was started 7 days later using eight week old AT1aR KO mice (The Jackson Laboratory), as described previously [5,6]. Drugs were given using a flexible tube (size M mouse Pro Funnel administration system, Torpac, NJ, USA) via pharynx of mouse. Mice were housed in the animal facilities of Osaka University with free access to food and water. Mice were euthanized at 4 weeks after treatment, before which BP was measured via the tail-cuff method.

\subsection{Materials}

Sac/Val was donated by Novartis International AG (Basel, Swiss). Valsartan was purchased from FUJIFILM Wako Pure Chemical Corporation (Osaka, Japan). All procedures were approved by the Animal Use and Care Committee of Osaka University. Systolic and diastolic BP were measured by the tail-cuff method (UR 5000: Softron, Tokyo, Japan), as described previously [7].

\subsubsection{Evaluation of Renal Fibrosis and Glomerular Injury}

At 35 days, the fibrosis was evaluated by Sirius red staining, and the glomeruli of the kidney were evaluated by Periodic Acid-Schiff (PAS) staining. To evaluate renal fibrosis, kidney sections (4 or 5 sections, $5 \mu$ m thick, per kidney) were stained with Sirius red staining and were analyzed visually under a light microscope by 2 investigators who were blinded to treatment. Sirius red-stained images were subsequently quantified using National Institutes of Health ImageJ [8]. To evaluate the glomerular injury, renal sections embedded in paraffin ( $5 \mu \mathrm{m}$ thick) were stained with PAS staining and examined by light microscopy. As described by [9], the glomerular injury scores were graded as $0: 0$ to $10 \% ; 1+$ : 10 to $25 \% ; 2+: 26$ to $50 \% ; 3+: 51$ to $75 \%$; and $4+: 75$ to $100 \%$. They were then analyzed visually under a light microscope by 2 investigators blinded to treatment. 


\subsubsection{Quantitative Real-time Polymerase Chain Reaction}

The total RNA was extracted from cultured cells, and quantitative real-time PCR was performed with an ABI Prism 7000 Sequence Detection System using PCR Master Mix Reagent (Applied Biosystems, Foster, USA). In each experiment, the mouse GAPDH RNA was amplified as a reference standard. Levels of mRNA were normalized to that of GAPDH mRNA, as described previously [10].

\section{STATISTICAL ANALYSIS}

Data are expressed as the mean \pm SEM. Comparisons were made using ANOVA followed by Turkey's simultaneous multiple comparisons. Values with $\mathrm{P}<0.05$ were considered significant.

\section{RESULTS}

\subsection{Evaluation for Renal Tubule Fibrosis by Sirius Red Staining}

In this study, we compared the effects of valsartan and Sac/Val on the mouse model of salt-sensitive hypertension using ATlaR-KO mice. First, we measured the systolic and diastolic BP. There was no significant difference among all groups before pretreatment. The treatment with only $1 \% \mathrm{NaCl}$ did not increase the systolic and diastolic BP, while treatment with valsartan as well as Sac/Val also did not decrease BP. Although the treatment with both $1 \% \mathrm{NaCl}$ and aldosterone infusion significantly increased systolic and diastolic BP $(\mathrm{P}<0.01)$, Sac/Val at a low- or high-dose treatment significantly decreased BP. The Sac/Val treatment also resulted in lower BP compared to valsartan at both low and high doses $(\mathrm{P}<0.01)$, (Fig. 1A , B).

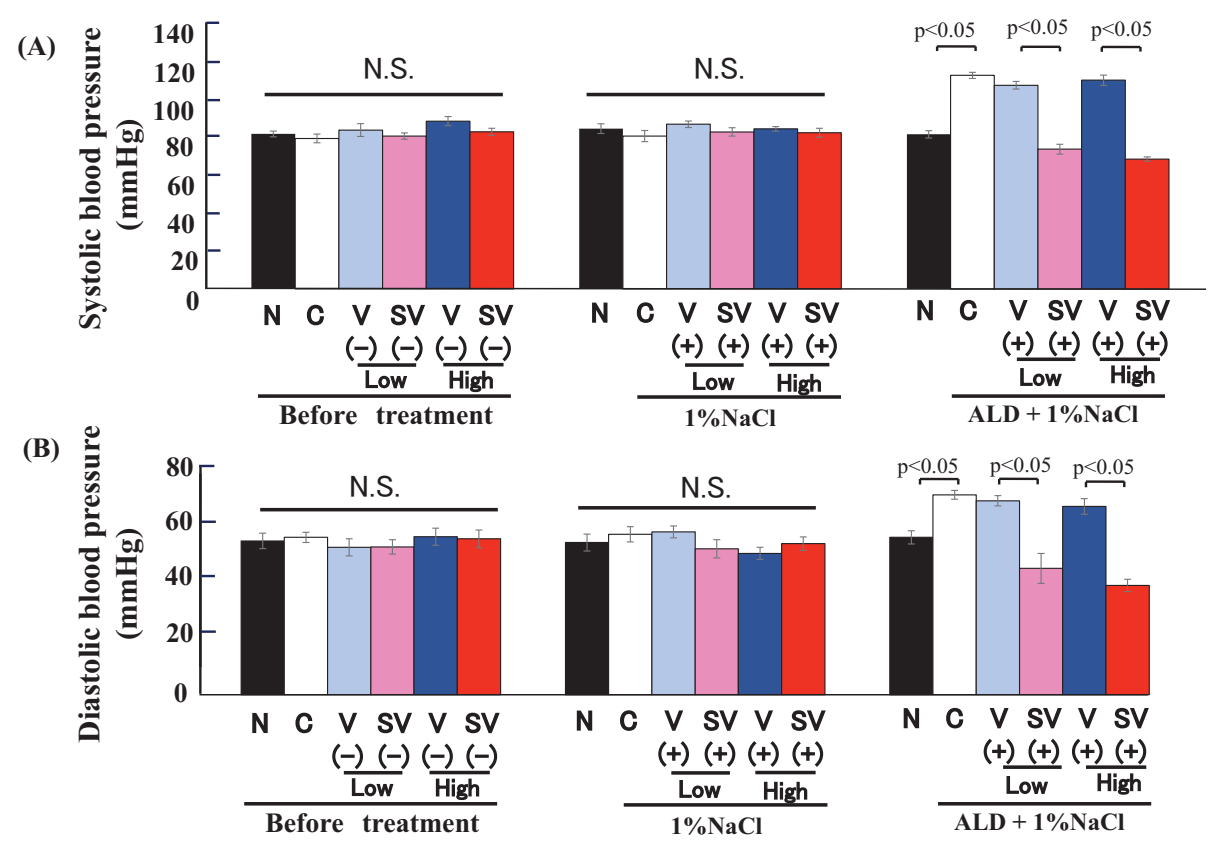

Fig. (1). \% Renal tubule fibrosis.

(A) The upper panel shows systolic blood pressure. Left graph; there is no significant change in systolic blood pressure before medication in AT1aR-KO mice. Middle graph; there is no significant change in systolic blood pressure after the Sac/Val (low dose $(30 \mathrm{mg} / \mathrm{kg} /$ day), high dose $(60 \mathrm{mg} / \mathrm{kg} /$ day)) or valsartan (low dose $(15 \mathrm{mg} / \mathrm{kg} /$ day), high dose $(30 \mathrm{mg} / \mathrm{kg} /$ day)) treatments. Right graph; after the treatment with aldosterone and $1 \% \mathrm{NaCl}$, systolic blood pressure is significantly increased $(\mathrm{p}<0.05)$, and $\mathrm{Sac} / \mathrm{Val}$ (low and high dose) significantly decreases the systolic blood pressure compared to valsartan (low and high dose) group (p $<0.05$ ).

(B) The lower panel shows diastolic blood pressure. Left graph; there is no significant change in diastolic blood pressure before treatment in ATlaR-KO mice. Middle graph; there is no significant change in diastolic blood pressure after Sac/Val (low dose (30 $\mathrm{mg} / \mathrm{kg} /$ day), high dose $(60 \mathrm{mg} / \mathrm{kg} /$ day)) or valsartan (low dose $(15 \mathrm{mg} / \mathrm{kg} /$ day), high dose $(30 \mathrm{mg} / \mathrm{kg} /$ day $))$ treatment. Right graph; after the treatment of aldosterone and $1 \% \mathrm{NaCl}$, diastolic blood pressure is significantly increased $(\mathrm{p}<0.05)$, and $\mathrm{Sac} / \mathrm{Val}(\mathrm{low}$ and high dose) significantly decreases the diastolic blood pressure compared to valsartan (low and high dose) group (p $<0.05$ ). N, Nontreatment; Con, Control; V, Valsartan; SV, Sacubitril/Valsartan; Low, low dose; High, high dose; NaCl, $1 \% \mathrm{NaCl}$; ALD, aldosterone. 
More importantly, the Sac/Val treatment, at both low and high doses, significantly decreased renal tubule fibrosis compared to the control $(\mathrm{P}<0.01$, Fig. 2), while aldosterone and the $1 \% \mathrm{NaCl}$ treatment significantly increased the renal tubule fibrosis area as evaluated by Sirius red staining in comparison to that of the non-treatment group (P<0.01).

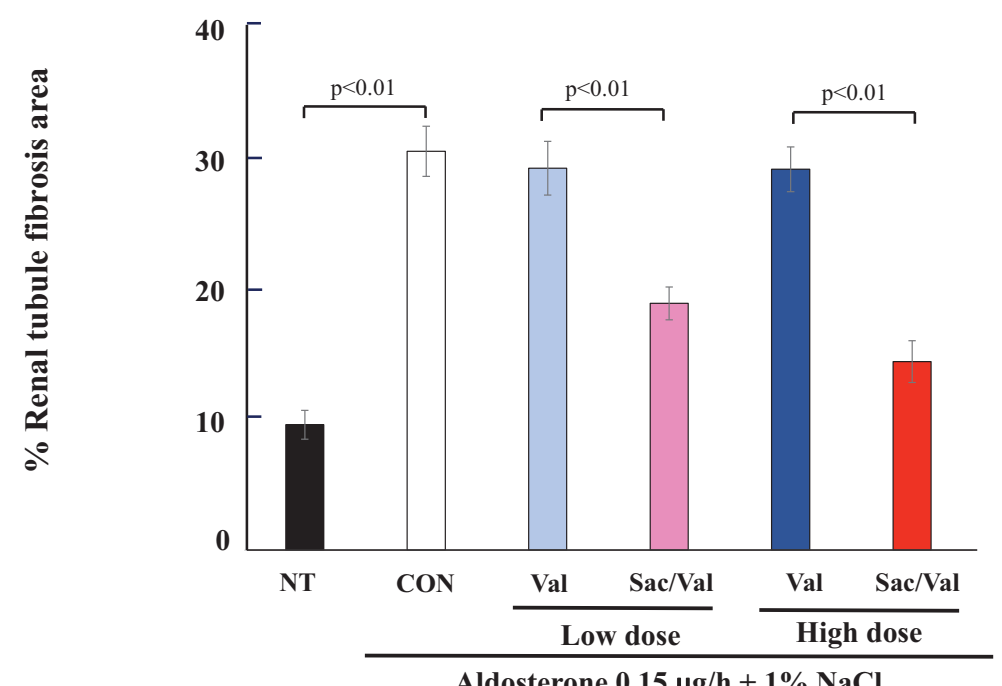

Fig. (2). \% Renal tubule fibrosis. We compared renal tubule fibrosis as evaluated by Sirius red staining. After the treatment with aldosterone and $1 \% \mathrm{NaCl}, \%$ renal tubule fibrosis is significantly increased $(\mathrm{p}<0.05)$, and $\mathrm{Sac} / \mathrm{Val}$ (low and high dose) significantly decreases \% renal tubule fibrosis compared to valsartan (low and high dose) $(\mathrm{p}<0.05)$. NT, non-treatment; CON, Control; Val, Valsartan; Sac/Val, Sacubitril/Valsartan.

\subsection{Evaluation for Glomerular Fibrosis by Sirius Red Staining and Glomerular Injury by PAS Staining}

Then, we further evaluated the area of renal glomerular fibrosis. As shown in Fig. (3), aldosterone and $1 \% \mathrm{NaCl}$ treatment significantly increased the glomerular fibrosis area as evaluated by Sirius red staining compared to the nontreatment group $(\mathrm{P}<0.01)$. Sac/Val treatment, both at low and high doses, significantly decreased glomerular fibrosis compared to valsartan in low- or high-dose treatments $(\mathrm{P}<0.01$, Fig. 3).
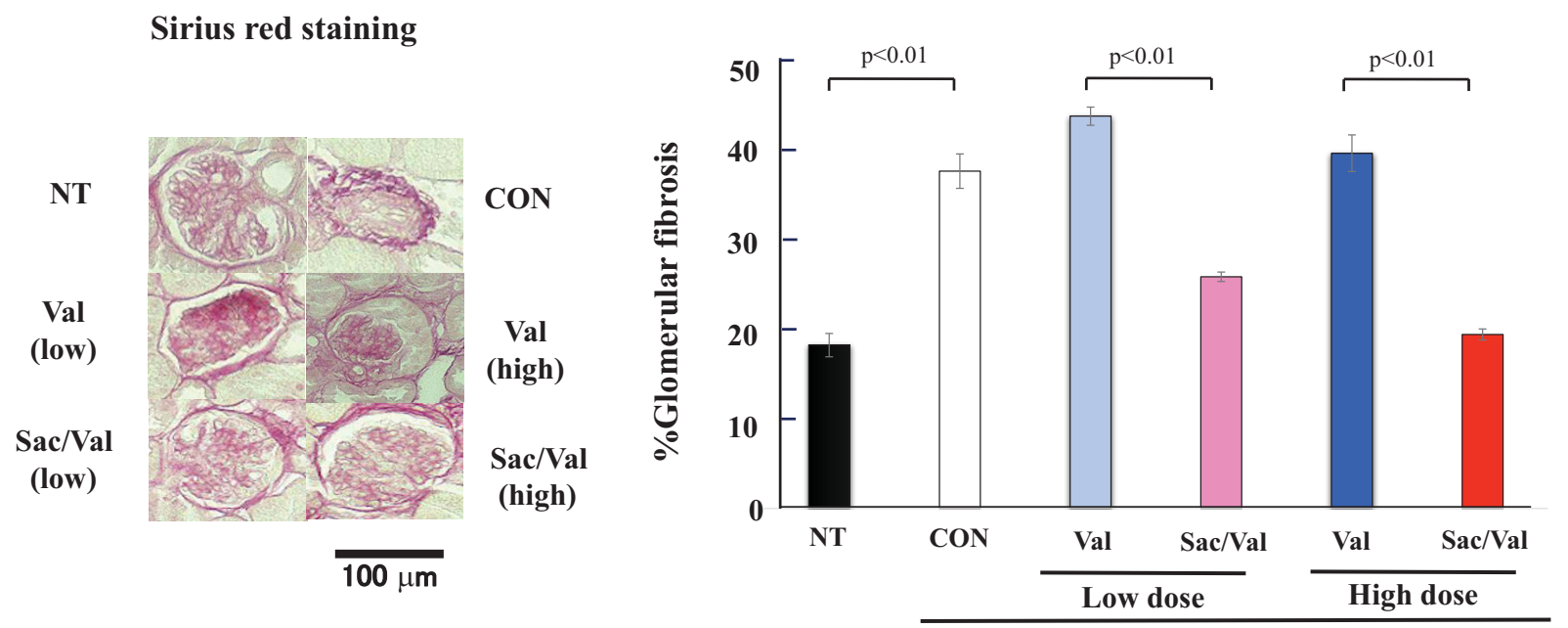

Aldosterone $0.15 \mu \mathrm{g} / \mathrm{h}+1 \% \mathrm{NaCl}$

Fig. (3). \% Glomerular fibrosis. We compared glomerular fibrosis as evaluated by Sirius red staining. After the treatment with aldosterone and $1 \% \mathrm{NaCl}, \%$ glomerular fibrosis is significantly increased $(\mathrm{p}<0.05)$, and $\mathrm{Sac} / \mathrm{Val}$ (low and high dose) significantly decreased \% glomerular fibrosis compared to valsartan (low and high dose) $(\mathrm{p}<0.05)$. NT; non-treatment, CON, Control; Val, Valsartan; Sac/Val, Sacubitril/Valsartan.

In addition, the aldosterone and $1 \% \mathrm{NaCl}$ treatment significantly increased the glomerular injury score as evaluated by PAS staining compared to no treatment $(\mathrm{P}<0.01)$. Similar to other evaluations, Sac/Val treatment at both low and 
high doses significantly decreased glomerular injury compared to valsartan low-and high-dose treatments $(\mathrm{P}<0.01$, Fig. 4).
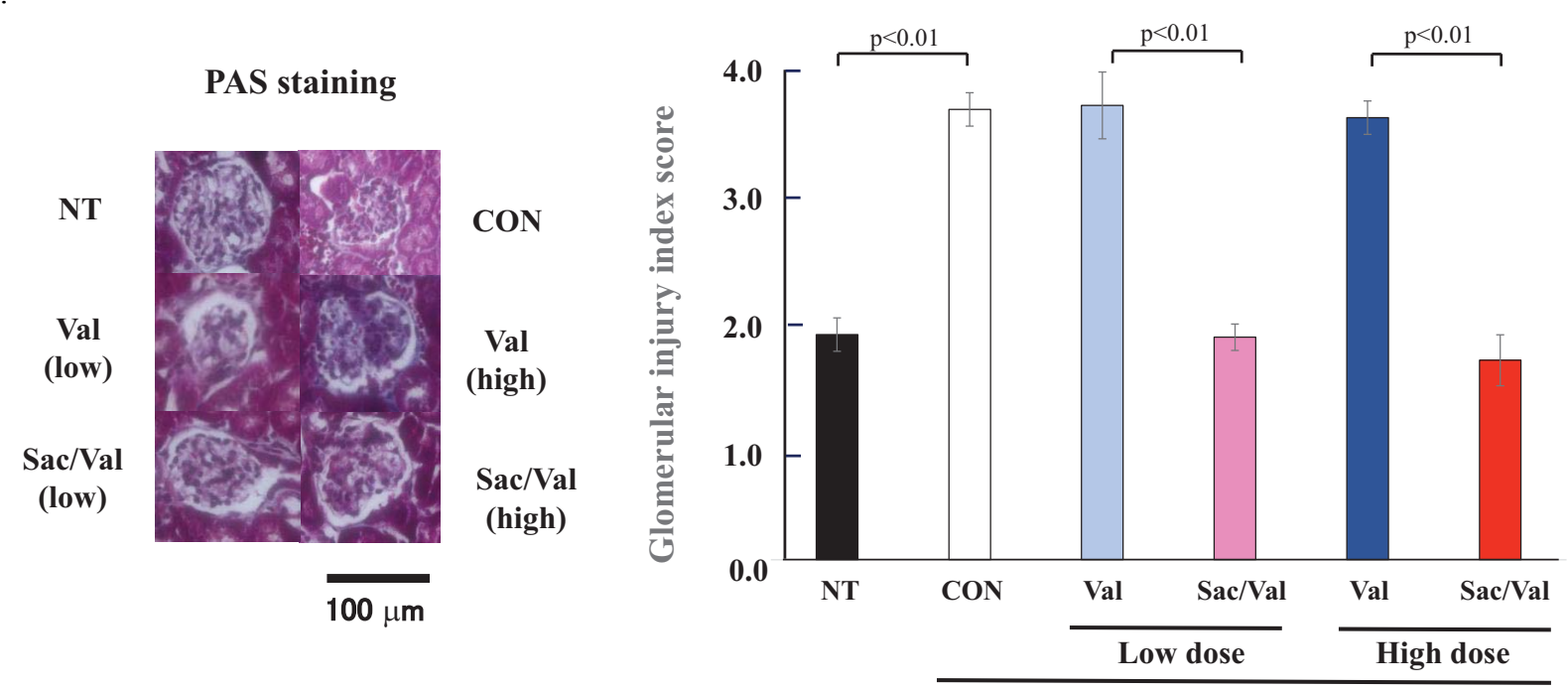

Aldosterone $0.15 \mu \mathrm{g} / \mathrm{h}+1 \% \mathrm{NaCl}$

Fig. (4). Glomerular injury. We compared glomerular injury as evaluated by PAS staining. After the treatment with aldosterone and $1 \% \mathrm{NaCl}$, the glomerular injury score is significantly increased $(\mathrm{p}<0.05)$, and Sac/Val; Sacubitril/Valsartan (low and high dose) significantly decreases the score compared to valsartan (low and high dose) $(\mathrm{p}<0.05)$. NT, non-treatment; CON, Control; Val, Valsartan; Sac/Val, Sacubitril/Valsartan.

\subsection{Evaluation for TGF- $\beta 1$ mRNA Synthesis by Real-time PCR}

Next, we further evaluated the TGF- $\beta 1$ mRNA synthesis of the kidney as evaluated by real-time PCR. As shown in Fig. (5), the aldosterone and $1 \% \mathrm{NaCl}$ treatment significantly increased the TGF- $\beta 1 \mathrm{mRNA}$ synthesis of the kidney compared to no treatment $(\mathrm{P}<0.01)$. Sac/Val treatment, in both low and high doses, significantly decreased TGF- $\beta 1$ mRNA synthesis compared to valsartan in low- or high-dose treatments $(\mathrm{P}<0.01$, Fig. 5).

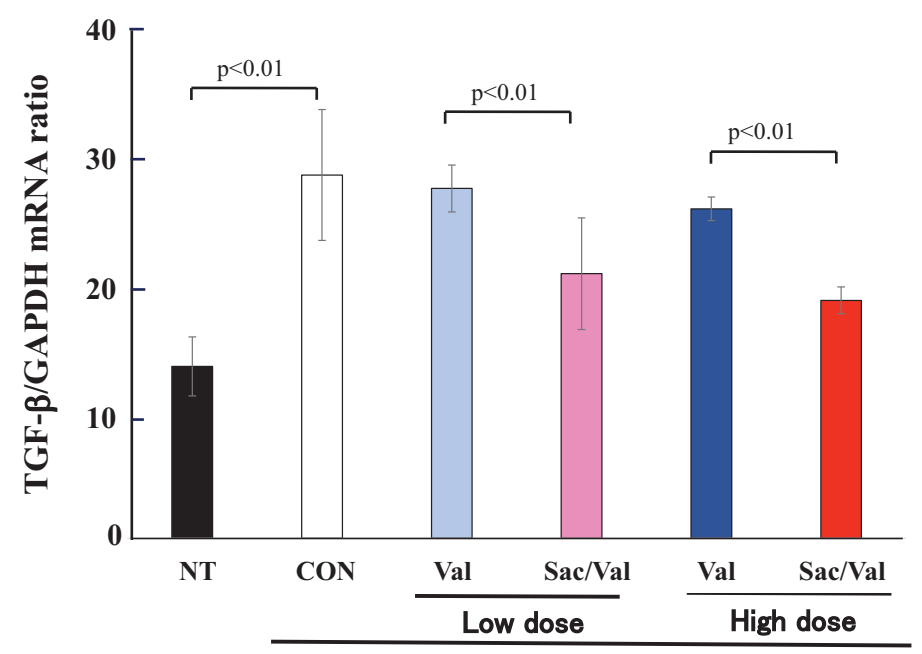

Aldosterone $0.15 \mu \mathrm{g} / \mathrm{h}+1 \% \mathrm{NaCl}$

Fig. (5). TGF- $\beta 1$ real-time PCR. We compared TGF- $\beta 1$ mRNA by real-time PCR. After the treatment with aldosterone and $1 \%$ $\mathrm{NaCl}$, TGF- $\beta 1$ mRNA is significantly increased $(\mathrm{p}<0.05)$, and Sac/Val; sacubitril/valsartan (low and high dose) significantly decreases TGF- $\beta 1$ mRNA compared to valsartan (low and high dose) $(\mathrm{p}<0.05)$. NT, non-treatment; CON, Control; Val, Valsartan; $\mathrm{Sac} / \mathrm{Val}$, Sacubitril/Valsartan.

\section{DISCUSSION}

The present study showed the additional inhibitory effects of sacubitril/valsartan on renal tubule fibrosis, glomerular 
fibrosis and injury compared to valsartan in a salt-sensitive hypertensive mouse model using AT1aR-KO mice. In these AT1aR-KO mice, the Ang II blockade had no effects on lowering BP or on organ protection. Indeed, valsartan not only decreased the systolic and diastolic BP but also affected the tubule fibrosis, glomerular fibrosis and injury that were induced by aldosterone treatment. Nevertheless, Sac/Val treatment resulted in a significant decrease in BP and organprotective actions on tubule fibrosis, glomerular fibrosis and injury. These data clearly demonstrated that Sac/Val likely has additional organ-protective actions, probably through the neprilysin inhibition independent from the Ang II blockade.

Did the reduction of blood pressure by Sac/Val improve renal injury? Ogawa et al. reported that hydralazine decreased the mice blood pressure induced by aldosterone and salt treatment, but it failed to improve renal fibrosis and glomerular damage [11]. The only effect of blood pressure reduction cannot improve renal fibrosis and glomerular damage in this model, so Sac/Val would improve them by the effect other than to lower the blood pressure.

Neprilysin is a ubiquitous enzyme that is responsible for the breakdown of many vasoactive peptides, including biologically active Natriuretic Peptides (NPs), adrenomedullin, substance P, bradykinin, endothelin-1, and angiotensin (Fig. 6). NPs are endogenous protein molecules that cause urinary sodium excretion. NPs consists of the three main NPs that are secreted from the heart: Atrial Natriuretic Peptide (ANP), B-type Natriuretic Peptide (BNP), and C-type Natriuretic Peptide (CNP) [12, 13]. While CNP from endothelial cells and cardiac fibroblasts do not exert marked effects on sodium or water excretion [14], ANP primarily secreted from the atria and BNP from the ventricles are circulating hormones that counteract the volume overloaded state by promoting natriuresis, diuresis, vasodilation, and Renin-Angiotensin System (RAS) blockade [15]. ANP and BNP decrease the preload via diuresis and afterload via vasodilation and RAS blockade. CNP acts locally and exhibits vasodilatory, antifibrotic and antihypertrophic effects on the myocardium [16]. CNP inhibits deoxyribonucleic acid and collagen synthesis of cardiac fibroblast, and its effect is stronger than ANP and BNP [17]. Moreover, CNP is locally secreted in the kidney as well as the heart or vessel [18], and CNP ameliorates acute kidney injury by inhibiting apoptosis and oxidative stress [19]. Overall, circulating ANP and BNP may improve renal damage through a decrease in BP by promoting natriuresis, diuresis, and vasodilation, and local CNP in the kidney may also improve the local renal damage. An increase in NPs caused by the inhibition of neprilysin by Sac/Val might contribute to the renal protective actions observed in this study.

Another possible explanation might be the contribution of adrenomedullin and bradykinin. Adrenomedullin is a potent vasodilator that reduces systemic vascular resistance, induces renal vasodilation, increases the glomerular blood flow and filtration rate, and sodium excretion [20]. Bradykinin is also a vasodilator for coronary, celiac, superior mesenteric, renal splenic, pulmonary, gastric, and femoral artery [21] and is exerted in the blood and in the kidney [22]. These vasodilators may contribute to the decrease in BP by Sac/Val. Importantly, in the phase III clinical trial ( $\mathrm{n}=1215$ ), Sac/Val significantly decreased systolic and diastolic BP and pulse pressure compared to the use of valsartan alone to treat patients with mild-to-moderate hypertension. Sac/Val was well tolerated, since no cases of angioedema were reported [23]. Thus, the result of this study is compatible with the clinical data.

In addition, in the impact on global mortality and morbidity in heart failure (PARADIGM-HF) trial, Sac/Val reduced cardiovascular and all-cause mortality, as well as HF hospitalizations, in patients with HFrEF compared to enalapril [4], although, the addition of enalapril to conventional therapy significantly reduced mortality and hospitalization for patients with HFrEF in the SOLVD trial [24]. Then, Sac/Val was approved for the treatment of patients with HFrEF in Europe and in the USA. The current ESC-HF guideline recommends Sac/Val to replace ACEI/ARB in ambulatory patients with HFrEF who remain symptomatic despite treatment with ACEIs, beta-blockers, and mineral corticoid antagonists (MRAs) [25], while an ACCF/AHA guideline update recommends Sac/Val in patients with chronic symptomatic HFrEF and New York Heart Association class II or III who can tolerate an ACEI or ARB [26]. In addition, Sac/Val also reduced NT-proBNP to a greater extent compared to enalapril in HF patients with the preserved Ejection Fraction (HFpEF) [27]. Moreover, in the PARAMOUNT trial, Sac/Val significantly reduced NTproBNP compared to valsartan in patients with HFpEF [28].

On the other hand, plasma aldosterone levels are known to be elevated in a subset of patients despite Ang II blockade therapy. This phenomenon, known as 'aldosterone breakthrough', is strongly associated with an increased risk of a declining glomerular filtration rate through the pro-fibrotic actions of aldosterone [2]. However, it is still unclear how Sac/Val prevented organ injury despite the Ang II blockade. To evaluate the molecular mechanisms for how $\mathrm{Sac} / \mathrm{Val}$ exhibited organ-protective actions, we focused on aldosterone breakthrough with long-term renin-angiotensin inhibition. Our present study might also provide an additional organ-protective episode of Sac/Val in aldosterone breakthrough, in addition to the clinical efficacy of Sac/Val. In this study, Sac/Val improved renal tubule fibrosis, 
glomerular fibrosis and injury induced by $1 \% \mathrm{NaCl}$ and aldosterone treatment compared to valsartan (Fig. 6).

In this study, we measured proteinuria in each mice, but we could not detect the difference between Sac/Val and valsartan. In addition, Jing $\mathrm{W}$ et al. demonstrated that there is significant renal histological and serum creatinine differences between Sac/Val and valsartan in 5/6 nephrectomy CKD rat model, but there is not a significant difference of $24 \mathrm{~h}$ proteinuria between them [29]. Moreover, in PARAMOUT trial, the estimated Glomerular Filtration Rate (eGFR) declined less in the Sac/Val than in the valsartan group $(-1.5$ vs. $-5.2 \mathrm{~mL} / \mathrm{min}$ per $1.73 \mathrm{~m}(2) ; \mathrm{P}=0.002)$ $\mathrm{Sac} / \mathrm{Val}$ is seemed to be better for renal function compared to valsartan in clinic [30]. In this experiment, we did not show the effect of renal function, but Sac/Val would improve it compared to valsartan.

In a study on the mechanism for why Sac/Val reduced renal fibrosis compared to valsartan alone, Suematsu et al. demonstrated that Sac/Val reduced cardiac fibrosis in diabetic mice through the suppression of TGF- $\beta$, but not MCP-1, IL-6, THF-a, and HIF-1a compared to the effects of valsartan [31]. The changes in those cytokines might contribute to the organ-protective actions of Sac/Val. In this study, Sac/Val treatment at both low and high doses significantly decreased TGF- $\beta$ mRNA synthesis of kidney compared to valsartan in low- or high-dose treatments. The Sac/Val treatment would decrease TGF- $\beta$ through neprilysin inhibition independent of an AT1R impediment. The further mechanism would be necessary in the future.

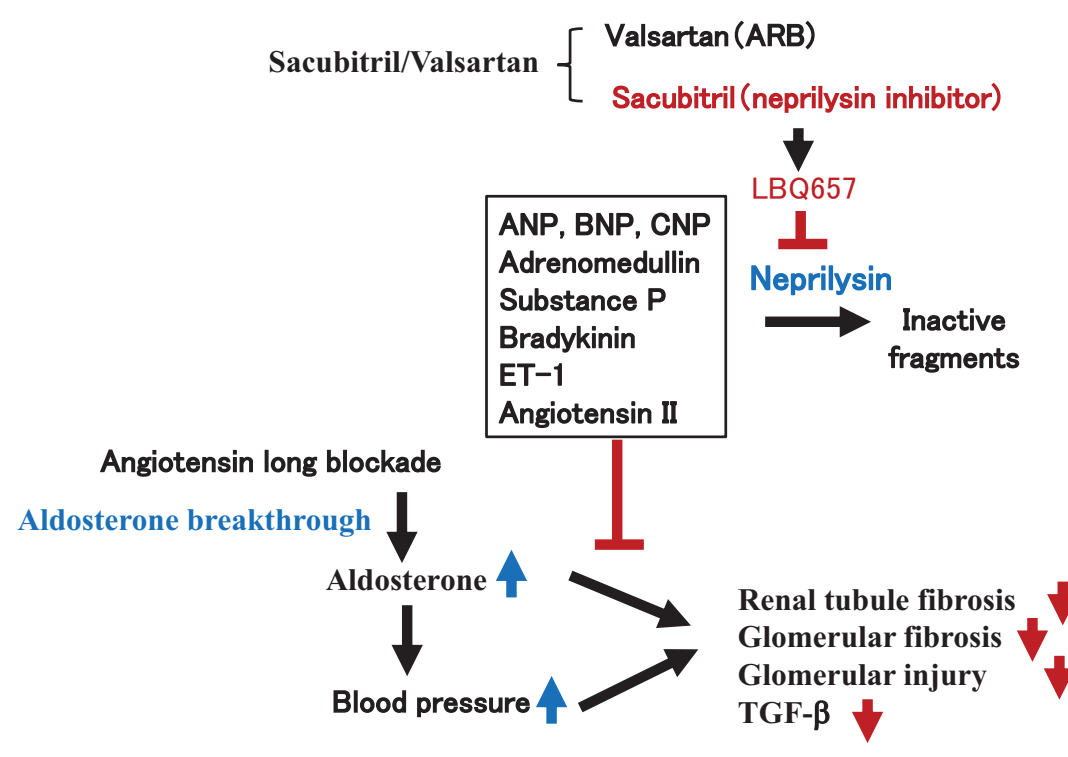

Fig. (6). Explanation. Sacubitril/valsartan contains valsartan and sacubitril (neprilysin inhibitor) $=$ LBQ657. Neprilysin changes ANP, BNP, CNP, adrenomedullin, substance P, bradykinin, angiotensin II, etc., into inactive fragments, whereas Sac/Val increases them. On the other hand, angiotensin long blockade induces aldosterone through the aldosterone breakthrough and increases the blood pressure. Increased aldosterone and blood pressure cause renal tubule fibrosis, glomerular fibrosis and injury, and sacubitril/valsartan decreases them.

In addition, Ougaard et al. demonstrated that use of high dose losartan $(30 \mathrm{mg} / \mathrm{kg})$ but not low dose losartan $(15 \mathrm{mg} / \mathrm{kg})$ decreased albumin creatinine ratio in Nephrotpxic Nephritis (NTN) mice CKD model [32]. In this experiment, there was a tendency that high dose of Sac/Val decreased more \% renal tubule fibrosis area and \% glomerular fibrosis area compared to low dose of that. High dose of Sac/Val may be better for reducing renal fibrosis like losartan.

\section{CONCLUSION}

The present study showed that Sac/Val exhibited more potent actions to decrease BP and inhibit renal injury and fibrosis than valsartan alone in salt sensitive hypertensive model using AT1aR-KO mice. Clearly, these additional beneficial effects of Sac/Val were due to non-angiotensin blockade, probably neprilysin inhibition. Sac/Val with the dual actions of AT1R blockade and neprilysin inhibition may provide more clinical valuable options for the treatment of hypertensive patients such as with aldosterone breakthrough. Although further studies are necessary. 


\section{LIMITATION}

At first, we cannot find the molecular mechanism how neprilysin inhibitor decreases renal fibrosis and injury. At least, TGF- $\beta 1$ reduction would decrease them, but we cannot conclude what decrease TGF- $\beta 1$ concretely through neprilysin inhibition. Further study is needed in future. Second, we don't check the upregulation of ANP, BNP, and CNP after Sac/Val treatment. Because the metabolism of them is too fast, we cannot detect them in the serum after drug treatment. Third, we use AT1aR KO, so AT1bR exists in mice. Valsartan (ARB) may inhibit AT1bR, but Valsartan doesn't decrease BP in AT1aR KO miec, so AT1bR may not effect on it too much. Fourth, Telemetry System is better to measure physiological blood pressure for mice, but the system is too expensive. In this experiment, we could not use it. We used the tail-cuff method to measure mice blood pressure, but there was a significant difference between Sac/Val group and valsartan group. I believe that this result has a meaning.

\section{LIST OF ABBREVIATIONS}

\begin{tabular}{|c|c|c|}
\hline AT1aR & $=$ & Angiotensin II type 1a Receptor \\
\hline BP & $=$ & Blood Pressure \\
\hline Ang II & $=$ & Angiotensin II \\
\hline ARBs & $=$ & Angiotensin II type 1 Receptor Blockers \\
\hline Sac/Val & $=$ & Sacubitril/Valsartan \\
\hline ARNI & $=$ & Angiotensin Receptor-Neprilysin Inhibitor \\
\hline NPs & $=$ & Natriuretic Peptides \\
\hline ET-1 & $=$ & Endothelin-1 \\
\hline HF & $=$ & Heart Failure \\
\hline HFrEF & $=$ & Heart Failure with Reduced Ejection Fraction \\
\hline ACEI & $=$ & Angiotensin Converting Enzyme Inhibitor \\
\hline NEPi & $=$ & Neprilysin Inhibitor \\
\hline PAS & $=$ & Periodic Acid-Schiff \\
\hline GAPDH & $=$ & Glyceraldehyde-3-phosphate Dehydrogenase \\
\hline TGF- $\beta 1$ & $=$ & Transforming Growth Factor Beta 1 \\
\hline $\mathbf{A N P}$ & $=$ & Atrial Natriuretic Peptide \\
\hline BNP & $=$ & B-type Natriuretic Peptide \\
\hline CNP & $=$ & C-type Natriuretic Peptide \\
\hline RAS & $=$ & Renin-Angiotensin System \\
\hline HFpEF & $=$ & Heart Failure with the Preserved Ejection Fraction \\
\hline MRAs & $=$ & Mineral Corticoid Antagonists \\
\hline МCP-1 & $=$ & Monocyte Chemotactic Protein-1 \\
\hline IL-6 & $=$ & Interleukin-6 \\
\hline TNF-a & $=$ & Tumor Necrosis Factor- $\alpha$ lpha \\
\hline HIF-1a & $=$ & Hypoxia-Inducible Factor-1 alpha \\
\hline AT1bR & $=$ & Angiotensin II type $1 \mathrm{~b}$ Receptor \\
\hline eGFR & $=$ & Estimated Glomerular Filtration Rate \\
\hline NTN & $=$ & Nephrotoxic Nephritis \\
\hline
\end{tabular}

\section{SOURCES OF FUNDING}

This work was partially supported by a grant from the Osaka Kidney Foundation, a Grant-in-Aid from the Organization for Pharmaceutical Safety and Research, a Grant-in-Aid from the Ministry of Public Health and Welfare, a Grant-in-Aid from Japan Promotion of Science, and through special coordination funds of the Ministry of Education, Culture, Sports, Science and Technology, of the Japanese Government. 


\section{ETHICS APPROVAL AND CONSENT TO PARTICIPATE}

All procedures were approved by the Animal Use and Care Committee of Osaka University.

\section{HUMAN AND ANIMAL RIGHTS}

No humans were involved in this study, the reported experiments on animals were in accordance with the standards set forth in the 8th Edition of Guide for the Care and Use of Laboratory Animals (http:// grants.nih.gov/grants/olaw/ Guide-for-thecare-and-use-of-laboratory-animals.pdf) published by the National Academy of Sciences.

\section{CONSENT FOR PUBLICATION}

Not applicable.

\section{CONFLICT OF INTEREST}

This study was financially supported by Novartis Pharma K.K., Japan. Sacubitril/Valsartan was provided by Novartis AG.

\section{ACKOWLEDGEMENTS}

We appreciate Miss Hitomi Sasaki for official support, and all of the members of the Clinical Gene Therapy group at the Osaka University Graduate School of Medicine, for much helpful discussion and technical support.

\section{SUPPLEMENTARY MATERIAL}

Supplementary material is available on the publishers Website along with the published article.

\section{REFERENCES}

[1] de Gasparo M, Catt KJ, Inagami T, Wright JW, Unger T. International union of pharmacology. XXIII. The angiotensin II receptors. Pharmacol Rev 2000; 52(3): 415-72. [PMID: 10977869]

[2] Bomback AS, Klemmer PJ. The incidence and implications of aldosterone breakthrough. Nat Clin Pract Nephrol 2007; 3(9): 486-92. [http://dx.doi.org/10.1038/ncpneph0575] [PMID: 17717561]

[3] Solomon SD, Rizkala AR, Gong J, et al. Angiotensin receptor neprilysin inhibition in heart failure with preserved ejection fraction: Rationale and design of the PARAGON-HF trial. JACC Heart Fail 2017; 5(7): 471-82. [http://dx.doi.org/10.1016/j.jchf.2017.04.013] [PMID: 28662936]

[4] McMurray JJV, Packer M, Desai AS, et al. Angiotensin-neprilysin inhibition versus enalapril in heart failure. N Engl J Med 2014; 371(11): 993-1004.

[http://dx.doi.org/10.1056/NEJMoa1409077] [PMID: 25176015]

[5] Kagiyama S, Matsumura K, Fukuhara M, Sakagami K, Fujii K, Iida M. Aldosterone-and-salt-induced cardiac fibrosis is independent from angiotensin II type 1a receptor signaling in mice. Hypertens Res 2007; 30(10): 979-89. [http://dx.doi.org/10.1291/hypres.30.979] [PMID: 18049031]

[6] Kusunoki H, Taniyama Y, Rakugi H, Morishita R. Cardiac and renal protective effects of irbesartan via peroxisome proliferator-activated receptor $\gamma$-hepatocyte growth factor pathway independent of angiotensin II Type 1a receptor blockade in mouse model of salt-sensitive hypertension. J Am Heart Assoc 2013; 2(2): e000103.

[http://dx.doi.org/10.1161/JAHA.113.000103] [PMID: 23608606]

[7] Taniyama Y, Morishita R, Nakagami H, et al. Potential contribution of a novel antifibrotic factor, hepatocyte growth factor, to prevention of myocardial fibrosis by angiotensin II blockade in cardiomyopathic hamsters. Circulation 2000; 102(2): 246-52. [http://dx.doi.org/10.1161/01.CIR.102.2.246] [PMID: 10889138]

[8] Kusunoki H, Taniyama Y, Azuma J, et al. Telmisartan exerts renoprotective actions via peroxisome proliferator-activated receptor$\gamma /$ hepatocyte growth factor pathway independent of angiotensin II type 1 receptor blockade Hypertension 2012; 59(2): 308-16. [http://dx.doi.org/10.1161/HYPERTENSIONAHA.111.176263] [PMID: 22252391]

[9] Raij L, Azar S, Keane W. Mesangial immune injury, hypertension, and progressive glomerular damage in Dahl rats. Kidney Int 1984; 26(2): 137-43. [http://dx.doi.org/10.1038/ki.1984.147] [PMID: 6239058]

[10] Sanada F, Taniyama Y, Iekushi K, et al. Negative action of hepatocyte growth factor/c-Met system on angiotensin II signaling via liganddependent epithelial growth factor receptor degradation mechanism in vascular smooth muscle cells. Circ Res 2009; 105(7): 667-75. [http://dx.doi.org/10.1161/CIRCRESAHA.109.202713] [PMID: 19713535]

[11] Ogawa Y, Mukoyama M, Yokoi H, et al. Natriuretic peptide receptor guanylyl cyclase-A protects podocytes from aldosterone-induced 
glomerular injury. J Am Soc Nephrol 2012; 23(7): 1198-209.

[http://dx.doi.org/10.1681/ASN.2011100985] [PMID: 22652704]

[12] Rosenzweig A, Seidman CE. Atrial natriuretic factor and related peptide hormones. Annu Rev Biochem 1991; 60(1): 229-55. [http://dx.doi.org/10.1146/annurev.bi.60.070191.001305] [PMID: 1652921]

[13] Yandrapalli S, Aronow WS, Mondal P, Chabbott DR. The evolution of natriuretic peptide augmentation in management of heart failure and the role of sacubitril/valsartan. Arch Med Sci 2017; 13(5): 1207-16. [http://dx.doi.org/10.5114/aoms.2017.68813] [PMID: 28883863]

[14] Taylor AL, Ziesche S, Yancy C, et al. Combination of isosorbide dinitrate and hydralazine in blacks with heart failure. N Engl J Med 2004; 351(20): 2049-57. [http://dx.doi.org/10.1056/NEJMoa042934] [PMID: 15533851]

[15] Levin ER, Gardner DG, Samson WK, Samson WK. Natriuretic peptides. N Engl J Med 1998; 339(5): 321-8. [http://dx.doi.org/10.1056/NEJM199807303390507] [PMID: 9682046]

[16] Soeki T, Kishimoto I, Okumura H, et al. C-type natriuretic peptide, a novel antifibrotic and antihypertrophic agent, prevents cardiac remodeling after myocardial infarction. J Am Coll Cardiol 2005; 45(4): 608-16. [http://dx.doi.org/10.1016/j.jacc.2004.10.067] [PMID: 15708711]

[17] Horio T, Tokudome T, Maki T, et al. Gene expression, secretion, and autocrine action of C-type natriuretic peptide in cultured adult rat cardiac fibroblasts. Endocrinology 2003; 144(6): 2279-84. [http://dx.doi.org/10.1210/en.2003-0128] [PMID: 12746286]

[18] Suzuki E, Hirata Y, Hayakawa H, et al. Evidence for C-type natriuretic peptide production in the rat kidney. Biochem Biophys Res Commun 1993; 192(2): 532-8. [http://dx.doi.org/10.1006/bbrc.1993.1448] [PMID: 8484764]

[19] Jin X, Zhang Y, Li X, Zhang J, Xu D. C-type natriuretic peptide ameliorates ischemia/reperfusion-induced acute kidney injury by inhibiting apoptosis and oxidative stress in rats. Life Sci 2014; 117(1): 40-5. [http://dx.doi.org/10.1016/j.1fs.2014.09.023] [PMID: 25283078]

[20] He H, Bessho H, Fujisawa Y, et al. Effects of a synthetic rat adrenomedullin on regional hemodynamics in rats. European Journal of Pharmacology 273(3): 209-14. 01995 [http://dx.doi.org/10.1016/0014-2999(94)00683-X]

[21] Cherry PD, Furchgott RF, Zawadzki JV, Jothianandan D. Role of endothelial cells in relaxation of isolated arteries by bradykinin. Proc Natl Acad Sci USA 1982; 79(6): 2106-10. [http://dx.doi.org/10.1073/pnas.79.6.2106] [PMID: 6952258]

[22] Campbell DJ, Kladis A, Duncan AM. Bradykinin peptides in kidney, blood, and other tissues of the rat. Hypertension (Dallas, Tex. : 1979). 1993; 21(2):155-165.

[http://dx.doi.org/10.1161/01.HYP.21.2.155]

[23] Ruilope LM, Dukat A, Böhm M, Lacourcière Y, Gong J, Lefkowitz MP. Blood-pressure reduction with LCZ696, a novel dual-acting inhibitor of the angiotensin II receptor and neprilysin: A randomised, double-blind, placebo-controlled, active comparator study. Lancet 2010; 375(9722): 1255-66.

[http://dx.doi.org/10.1016/S0140-6736(09)61966-8] [PMID: 20236700]

[24] Yusuf S, Pitt B, Davis CE, Hood WB, Cohn JN. Effect of enalapril on survival in patients with reduced left ventricular ejection fractions and congestive heart failure. N Engl J Med 1991; 325(5): 293-302.

[http://dx.doi.org/10.1056/NEJM199108013250501] [PMID: 2057034]

[25] Ponikowski P, Voors AA, Anker SD, et al. 2016 ESC guidelines for the diagnosis and treatment of acute and chronic heart failure: The task force for the diagnosis and treatment of acute and chronic heart failure of the European Society of Cardiology (ESC) developed with the special contribution of the Heart Failure Association (HFA) of the ESC. Eur Heart J 2016; 37(27): 2129-200. [http://dx.doi.org/10.1093/eurheartj/ehw128] [PMID: 27206819]

[26] Yancy CW, Jessup M, Bozkurt B, et al. 2016 ACC/AHA/HFSA focused update on new pharmacological therapy for heart failure: An update of the $2013 \mathrm{ACCF} / \mathrm{AHA}$ guideline for the management of heart failure: A report of the american college of cardiology/american heart association task force on clinical practice guidelines and the heart failure society of america. Circulation 2016; 134(13): e282-93. [PMID: 27208050]

[27] Packer M, McMurray JJV, Desai AS, et al. PARADIGM-HF investigators and coordinators. Angiotensin receptor neprilysin inhibition compared with enalapril on the risk of clinical progression in surviving patients with heart failure. Circulation 2015; 131(1): 54-61. [http://dx.doi.org/10.1161/CIRCULATIONAHA.114.013748] [PMID: 25403646]

[28] Solomon SD, Zile M, Pieske B, et al. The angiotensin receptor neprilysin inhibitor LCZ696 in heart failure with preserved ejection fraction: A phase 2 double-blind randomised controlled trial. Lancet 2012; 380(9851): 1387-95. [http://dx.doi.org/10.1016/S0140-6736(12)61227-6] [PMID: 22932717]

[29] Jing W, Vaziri ND, Nunes A, et al. LCZ696 (Sacubitril/valsartan) ameliorates oxidative stress, inflammation, fibrosis and improves renal function beyond angiotensin receptor blockade in CKD. Am J Transl Res 2017; 9(12): 5473-84.

[PMID: 29312499] 
[30] Voors AA, Gori M, Liu LCY, et al. Renal effects of the angiotensin receptor neprilysin inhibitor LCZ696 in patients with heart failure and preserved ejection fraction. Eur J Heart Fail 2015; 17(5): 510-7. [http://dx.doi.org/10.1002/ejhf.232] [PMID: 25657064]

[31] Suematsu Y, Miura S, Goto M, et al. LCZ696, an angiotensin receptor-neprilysin inhibitor, improves cardiac function with the attenuation of fibrosis in heart failure with reduced ejection fraction in streptozotocin-induced diabetic mice. Eur J Heart Fail 2016; 18(4): 386-93. [http://dx.doi.org/10.1002/ejhf.474] [PMID: 26749570]

[32] Ougaard ME, Jensen HE, Thuen ID, Petersen EG, Kvist PH. Inhibitors of the renin-angiotensin system ameliorates clinical and pathological aspects of experimentally induced nephrotoxic serum nephritis. Ren Fail 2018; 40(1): 640-8.

[http://dx.doi.org/10.1080/0886022X.2018.1533867] [PMID: 30403908]

\section{(C) 2018 Otsu et al.}

This is an open access article distributed under the terms of the Creative Commons Attribution 4.0 International Public License (CC-BY 4.0), a copy of which is available at: https://creativecommons.org/licenses/by/4.0/legalcode. This license permits unrestricted use, distribution, and reproduction in any medium, provided the original author and source are credited. 